\title{
Sub-fF-Capacitance Photonic-Crystal Photodetector Towards fJ/bit On-Chip Receiver
}

\author{
Kengo NOZAKI ${ }^{\dagger, \dagger \dagger a)}$, Nonmember, Shinji MATSUO ${ }^{\dagger, \dagger \dagger}$, Koji TAKEDA $^{\dagger, \dagger \dagger}$, Members, Takuro FUJII ${ }^{\dagger, \dagger \dagger}$, \\ Masaaki ONO ${ }^{\dagger, \dagger \dagger}$, Abdul SHAKOOR ${ }^{\dagger, \dagger \dagger}$, Eiichi KURAMOCHI ${ }^{\dagger, \dagger \dagger}$, and Masaya NOTOMI ${ }^{\dagger, \dagger \dagger}$, Nonmembers
}

\begin{abstract}
SUMMARY An ultra-compact InGaAs photodetector (PD) is demonstrated based on a photonic crystal $(\mathrm{PhC})$ waveguide to meet the demand for a photoreceiver for future dense photonic integration. Although the PhC-PD has a length of only $1.7 \mu \mathrm{m}$ and a capacitance of less than $1 \mathrm{fF}$, a high responsivity of $1 \mathrm{~A} / \mathrm{W}$ was observed both theoretically and experimentally. This low capacitance PD allows us to expect a resistor-loaded receiver to be realized that requires no electrical amplifiers. We fabricated a resistor-loaded PhC-PD for light-to-voltage conversion, and demonstrated a $\mathrm{kV} / \mathrm{W}$ efficiency with a $\mathrm{GHz}$ bandwidth without using amplifiers. This will lead to a photoreceiver with an ultralow energy consumption of less than $1 \mathrm{fJ} / \mathrm{bit}$, which is a step along the road to achieving a dense photonic network and processor on a chip.

key words: photonic crystal waveguide, photodetector, buried heterostructure, optical interconnection
\end{abstract}

\section{Introduction}

A highly integrated chip-scale photonic interconnection and/or a photonic-network-on-chip architecture are demanded for future microprocessors [1]-[3]. As the communication distance decreases and the integration density increases, the size and energy consumption of opto-electronic devices will become a significant issue, and so nanophotonic technologies should be implemented in the system. A photoreceiver will be a critical device because of its large energy consumption. This mainly originates from the need to use a trans-impedance amplifier (TIA) and some voltage amplifiers for a conventional receiver circuit, which incurs an energy cost of hundred $\mathrm{fJ} /$ bit and requires an area of more than $10^{4} \mu \mathrm{m}^{2}$ [4]-[6]. Therefore, this will constitute a significant bottleneck when implemented for dense on-chip communication [7]. One solution is to realize ultrasmallcapacitance photodetectors (PDs) and thus allow connection to a high impedance receiver circuit while maintaining a large resistance-capacitance (RC) bandwidth. This would lead to a reduction in electrical amplification or even its elimination (known as a receiver-less PD [5], [7], [8]), which promises an ultralow-energy on-chip photoreceiver.

Photonic crystals (PhCs) are promising for creating an

Manuscript received February 13, 2017.

Manuscript revised May 26, 2017.

${ }^{\dagger}$ The authors are with Nanophotonics Center, NTT Corporation, Atsugi-shi, 243-0198 Japan.

${ }^{\dagger}$ The authors are with NTT Basic Research Laboratories, NTT Corporation, Atsugi-shi, 243-0198 Japan.

${ }^{\dagger \dagger}$ The authors are with NTT Device Technology Laboratories, NTT Corporation, Atsugi-shi, 243-0198 Japan.

a) E-mail: nozaki.kengo@ lab.ntt.co.jp

DOI: $10.1587 /$ transele.E100.C.750 ultrasmall PD because of their strong light confinement. We have been studying such a PhC-PD with a compact InGaAs absorber embedded in an InP-based $\mathrm{PhC}$ waveguide, which we fabricated using a buried-heterostructure $(\mathrm{BH})$ formation technique [9]-[11]. This compact $\mathrm{BH}$ with a $\mathrm{PhC}$ waveguide can confine both photons and carriers in an ultrasmall space to achieve both high optical responsivity and ultrasmall device capacitance thus making it desirable in terms of meeting the demand for an amplifier-less photoreceiver.

In this paper, we describe an InGaAs-embedded PhCPD where the absorber length was reduced to only $1.7 \mu \mathrm{m}$ and the device capacitance was less than $1 \mathrm{fF}$. The size is still sufficient to obtain a high responsivity of $1 \mathrm{~A} / \mathrm{W}$ and a clear eye opening for a 40-Gbit/s signal. Furthermore, we fabricated a PhC-PD integrated with a several$\mathrm{k} \Omega$ load resistor to demonstrate on-chip light-to-voltage conversion. We employed an electro-optic (EO) probe to measure the voltage generation in the PhC-PDs, which revealed a conversion efficiency of as high as $4 \mathrm{kV} / \mathrm{W}$. The expected bandwidth after removing the parasitic wiring elements exceeds $10 \mathrm{GHz}[10]$. This suggests that the optical energy required for generating a CMOS voltage level is less than $1 \mathrm{fJ} / \mathrm{bit}$, which can be obtained without electrical amplification and therefore dominates the total energy consumption. These results reveal a successful way of realizing an ultrasmall/ultralow-energy photoreceiver that can be densely integrated on a chip.

\section{Requirement for Resistor-Loaded Photoreceiver}

To discuss the required capacitance of a PD used for configuring a resistor-loaded photoreceiver, we considered the simple PD-resistor circuit shown in Fig. 1 (a). This consists of PD capacitance $C_{\mathrm{j}}$, PD series resistance $R_{\mathrm{s}}$, load resistance $R_{\text {load }}$, and front-end capacitance (including parasitic capacitances) $C_{\mathrm{p}}$. The output voltage $V_{\text {out }}$ for the photocurrent $I_{\mathrm{pd}}$ is given by

$$
V_{\text {out }}=\frac{R_{\text {load }} I_{\mathrm{PD}}}{1-R_{\text {load }} R_{\mathrm{s}} C_{\mathrm{j}} C_{\mathrm{p}} \omega^{2}+j \omega\left[R_{\text {load }}\left(C_{\mathrm{j}}+C_{\mathrm{p}}\right)+R_{\mathrm{s}} C_{\mathrm{j}}\right]}
$$

For a low frequency response, $V_{\text {out }}=R_{\text {load }} I_{\mathrm{PD}}$, which leads to a transimpedance gain of $V_{\text {out }} / I_{\mathrm{PD}}=R_{\text {load }}$. The light-tovoltage conversion efficiency would be $\eta_{\mathrm{LV}}=V_{\text {out }} / P_{\text {opt }}=$ $\eta_{\text {pd }} R_{\text {load }}[\mathrm{V} / \mathrm{W}]$, where $\eta_{\text {pd }}[\mathrm{A} / \mathrm{W}]$ is optical responsivity. On 
(a)
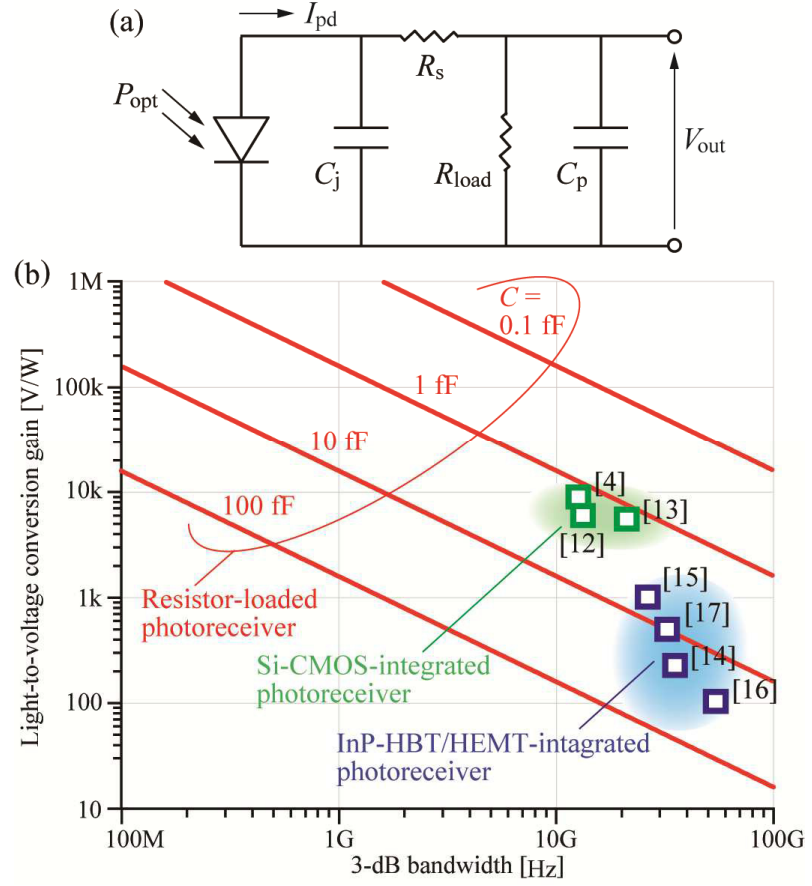

Fig. 1 Theoretical required capacitance for a resistor-loaded PD. (a) Equivalent circuit of the resistor-loaded PD. (b) Calculated light-tovoltage conversion efficiency and $3-\mathrm{dB}$ bandwidth (red lines). The total capacitance is changed as a parameter. The reported values for the Si-CMOS integrated photoreceivers (green plots) and the InP-HBT/HEMT-integrated photoreceivers (blue plots) are also plotted.

the other hand, the RC bandwidth $f_{\mathrm{RC}}$ can be obtained by setting the magnitude of the denominator of Eq. (1) at $\sqrt{ } 2$. Roughly speaking, the second term of the denominator is negligible below the $3-\mathrm{dB}$ bandwidth, and $R_{\mathrm{s}}$ is assumed to be much lower than $R_{\text {load }}$. As a result, $f_{\mathrm{RC}}$ is given by

$$
f_{\mathrm{RC}} \approx \frac{1}{2 \pi R_{\text {load }}\left(C_{\mathrm{j}}+C_{\mathrm{p}}\right)}
$$

Figure 1 (b) shows the calculated light-to-voltage conversion efficiency and the corresponding 3-dB bandwidth. Bandwidth limit due to the carrier transit time is not included in this calculation. The total capacitance $C=$ $C_{\mathrm{j}}+C_{\mathrm{p}}$ is changed as a parameter, which clearly determines the efficiency-bandwidth product (EBP) as given by $\eta_{\mathrm{LV}} f_{\mathrm{RC}}=\eta_{\mathrm{pd}}(2 \pi C)^{-1}$. We assume an optical responsivity $\eta_{\mathrm{pd}}=1 \mathrm{~A} / \mathrm{W}$, which is actually obtained in the experiment. Figure 1 (b) also includes plots for reported Ge-based photoreceivers integrated with a Si-CMOS TIA/Limiting amplifier (LA) circuit [4], [12], [13] and InGaAs-based photoreceivers integrated with an InP-based heterojunction bipolar transistor (HBT)/high electron mobility transistor (HEMT) circuit [14]-[17]. These receivers support a high conversion efficiency and large bandwidth thanks to the integrated amplifiers. However, it should be noted that such amplifiers also consume a huge amount of electric power (several $\mathrm{mW}$ ) [5], [6], and this dominates the overall power consumption. To exceed these conventional receivers with resistor-loaded receiver in terms of EBP, $C$ should be lower than $1 \mathrm{fF}$. Recent Ge-waveguide PDs have exhibited a capacitance of 4-5 fF [18], [19], which does not meet this requirement.

A low-capacitance resistor-loaded receiver also has the advantage on the signal-to-noise ratio because of its high resistance and consequent low thermal noise even at a $\mathrm{GHz}$ range. For the amplifier-integrated receiver, the amplifier noise generally limits the lowest optical power required for the error-free operation. For the resistor-loaded receiver, on the other hand, the required voltage at the front end basically determines the optical power. At this situation, the noise level is enough low for the error-free operation [10].

To these purposes, we need to focus on reducing the PD capacitance to achieve a resistor-loaded receiver that offers sufficiently high performance without the need for any signal amplifiers.

\section{Ultra-Small Capacitance Photonic-Crystal PD}

\subsection{Device Structure and Capacitance}

We have employed the combination of a $\mathrm{PhC}$ waveguide and cavity and an ultrasmall $\mathrm{BH}$ to demonstrate optical nanodevices such as nanolasers and all-optical memories [9], [11], which exhibited a record low power consumption. This structure can be employed for PDs by embedding a compact InGaAs absorber, thereby reducing the device capacitance by miniaturization. Figure 2 (a) shows a schematic of our PhC-PD. The device consists of an InP PhC waveguide, a $\mathrm{BH}$ for embedding the InGaAs, and a lateral p-i-n junction [20]. The inset shows the fundamental waveguide mode profile, which was simulated with the finite-element method (FEM). The lattice constant $a$ and the air-hole diameter $2 r$ were 420 and $200 \mathrm{~nm}$, respectively $(2 r / a=0.476)$. The InP slab and the InGaAs absorber were 250 and $150 \mathrm{~nm}$ thick, respectively, and the InGaAs was $350 \mathrm{~nm}$ wide. The waveguide width was set at $0.95 W_{0}$, where $W_{0}$ is the basic line defect width defined as the removal of one row of air holes (W1 waveguide). The optical confinement factor $(\Gamma)$ in the embedded InGaAs absorber is given by

$$
\int_{\text {absorber }} \varepsilon|E|^{2} d \mathbf{r} / \int_{A l l} \varepsilon|E|^{2} d \mathbf{r}
$$

The propagation mode is concentrated in the waveguide core region at $a / \lambda=0.28$, thus resulting in a $\Gamma$ of as high as 0.5 . The group index $n_{\mathrm{g}}$ at this wavelength is 5 . The $\mathrm{PhC}$ waveguide also has a large $n_{\mathrm{g}}$ (slow light), which can lead to absorption enhancement. However, the slow-light waveguide needs an optimized coupler to prevent any significant optical reflection, and this is a topic for future study. As shown in this paper, the normal $n_{\mathrm{g}}$ value $(=5)$ still has good capability for achieving an ultrashort PD.

The small dimensions of the absorber and the p-i-n junction mean that the device capacitance should be reduced to the fF level, as shown in Fig. 2(b). The parallel-plate capacitance $\left(=\varepsilon_{0} \varepsilon_{\text {InGaAs }} L_{\mathrm{abs}} T_{\mathrm{j}} / d_{\mathrm{j}}\right)$ is less than $0.2 \mathrm{fF}$ for an absorber length $L_{\mathrm{abs}}<3 \mu \mathrm{m}$. However, for an ultrasmall 
(a)

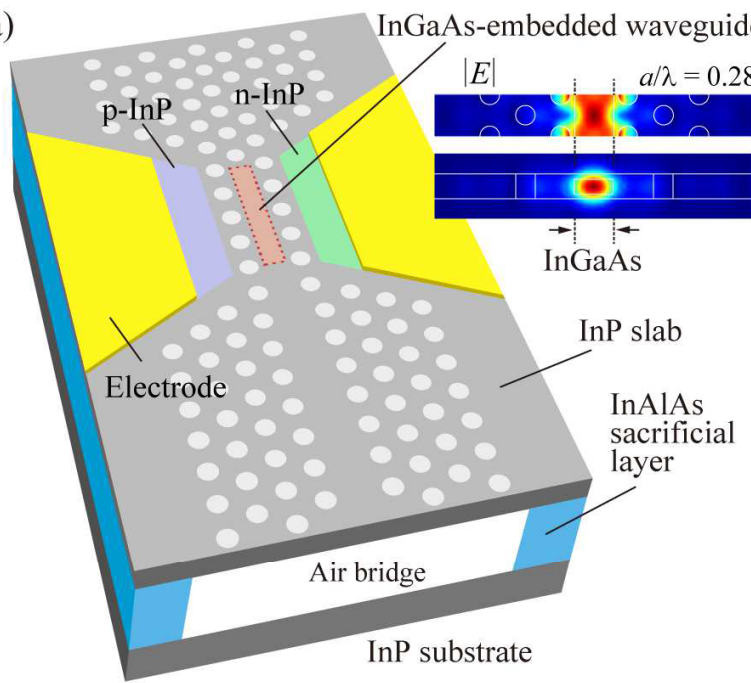

(b)

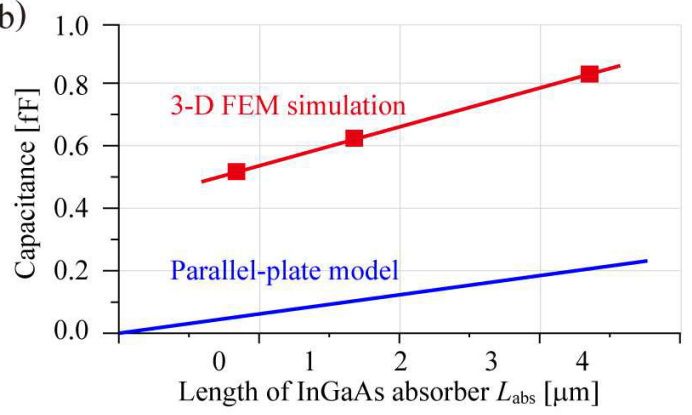

Fig. 2 PhC-PD structure and capacitance. (a) Schematic of PhC-PD. The inset is the calculated mode profile of the fundamental mode. (b) Calculated capacitance of PhC-PD. The blue curve is calculated from the parallel-plate model. The red plots are the results simulated by FEM with a 3-D model.

junction, the fringing field contribution of the junction also becomes significant, and hence it is important to include the fringe capacitance [21]. This contribution was simulated by the FEM with a full 3-D model excluding the large electrical pads, and the total capacitance exceeded that of the parallel plate model. The total capacitance of our PhC-PD is still $<1 \mathrm{fF}$, which is smaller than our target given the goal of realizing a resistor-loaded receiver, as discussed in the Sect. 2.

\subsection{Responsivity Calculation}

We simulated the optical responsivity for this PhC-PD using the commercial software (Lumerical [22]). A 3-D finitedifference time domain (FDTD) solver was used to simulate the light propagation and absorption, while an FEM-based carrier transport solver was used to calculate the responsivity. Figure 3 (a) shows the simulation model, consisting of an InP PhC and an embedded InGaAs absorber. An absorber length $L_{\text {abs }}$ of $4 \mathrm{a}$, and an absorption coefficient of $10^{4} \mathrm{~cm}^{-1}$ (imaginary part of index $k=0.124$ ) were assumed. Other parameters are the same as in Fig. 2(a). Because of the index difference between the InP PhC waveguide and the InGaAs-embedded waveguide, their widths were adjusted so that their guiding bands matched. To this end, the widths

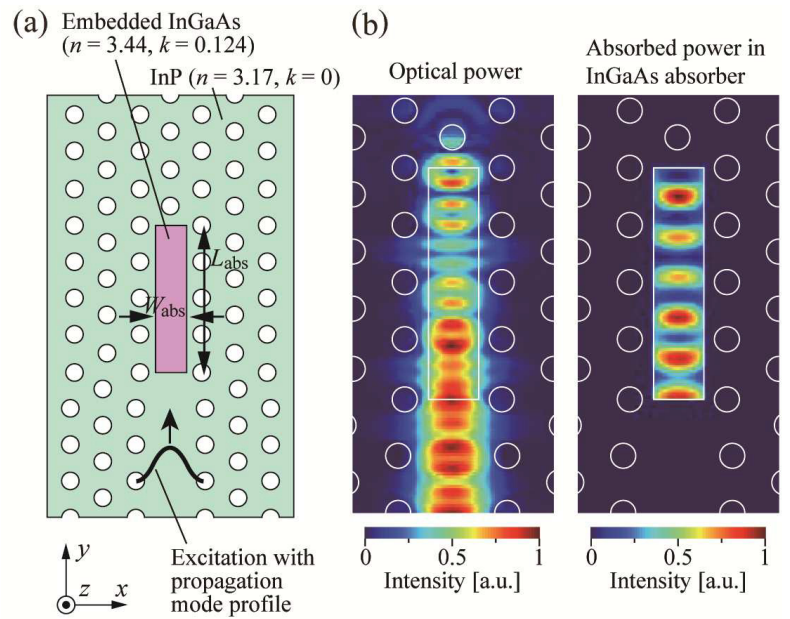

(c)
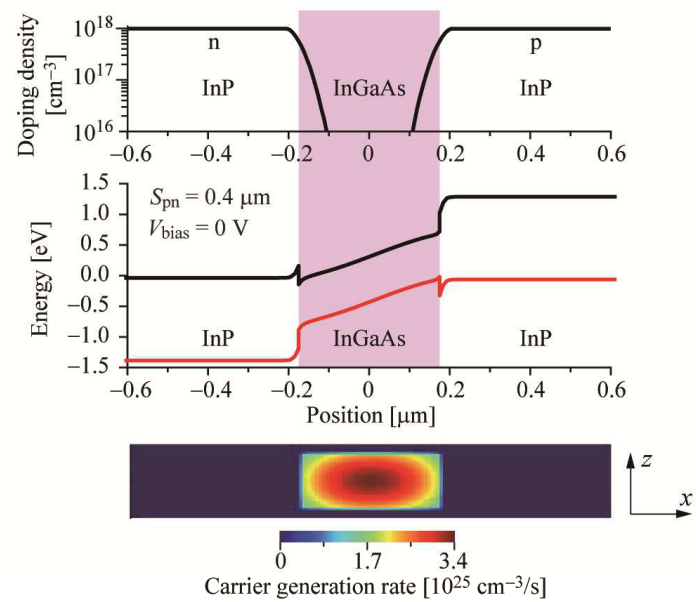

(d)

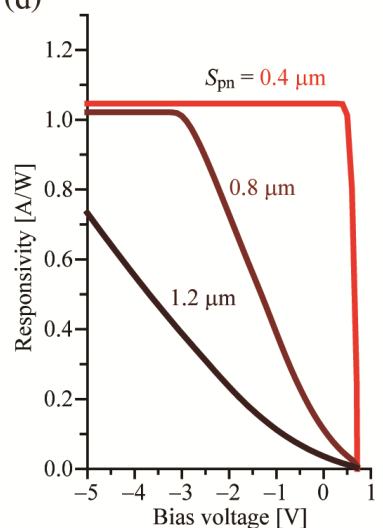

(e)

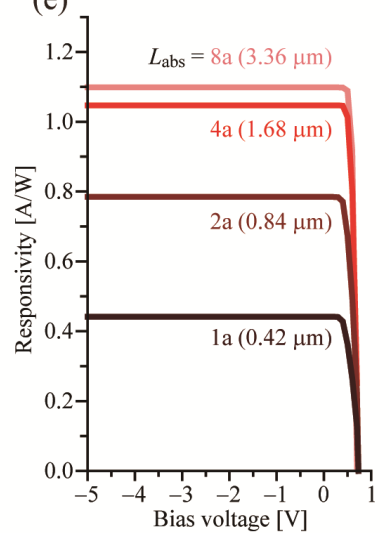

Fig.3 Calculated responsivity of PhC-PD. (a) Calculation model for $L_{\mathrm{abs}}=4 a(1.68 \mu \mathrm{m})$ and $W_{\mathrm{abs}}=350 \mathrm{~nm}$. (b) Propagated light power profile (left) and absorbed power profile (right). (c) Doping profile (top), energy band diagram (middle), and carrier generation rate profile for incident optical power of $1 \mu \mathrm{W}$. (d) Calculated responsivity for different $\mathrm{p} / \mathrm{n}$ separations $S_{\mathrm{pn}} . L_{\mathrm{abs}}=4 a$ is fixed. (e) Calculated responsivity for different $L_{\mathrm{abs}}$. $S_{\mathrm{pn}}=0.4 \mu \mathrm{m}$ is fixed.

of the InP and InGaAs-embedded regions were changed to $1.1 W_{0}$ and $0.95 W_{0}$, respectively. Excited light with the shape of the fundamental mode was introduced at the in- 
put waveguide to be forwarded to the absorber. Figure 3 (b) shows the power profiles of the propagated and absorbed light. We confirmed that most of the light was absorbed after a single roundtrip in the absorber, resulting in an absorption efficiency of $85 \%$.

Figure 3 (c) shows the cross-sectional doping profile and the corresponding energy band diagram that we used for the carrier transport simulation. The doping levels for both the $\mathrm{p}$ and $\mathrm{n}$ layers were $10^{18} \mathrm{~cm}^{-3}$ and the separation between them was $S_{\mathrm{pn}}=0.4 \mu \mathrm{m}$. The light absorption profile in Fig. 3(b) gives the carrier generation rate when the incident light power was set at $1 \mu \mathrm{W}$ in this calculation. To avoid a long calculation time, a 2-D carrier transport simulation was carried out, for which the optical absorption profile was averaged in the $y$ direction and thus the carrier generation rate profile on the $x-z$ axis was obtained as shown in Fig. 3 (c). Figure 3 (d) shows the calculated responsivity for the bias voltage when $S_{\mathrm{pn}}$ was changed. When $S_{\mathrm{pn}}=0.4$ $\mu \mathrm{m}$, which matches the absorber width, a flat responsivity of $1.02 \mathrm{~A} / \mathrm{W}$ was calculated for the entire reverse voltage range. Increases in $S_{\text {pn }}$ make the static electric field weaker, resulting in carrier accumulation and poor carrier extraction compared with carrier recombination (a lifetime of $10 \mathrm{~ns}$ in this calculation). This suggested that the PhC-PD would maintain a high responsivity thanks to the narrow optical confinement and subsequent local absorption. On the other hand, Fig. 3 (d) shows the responsivities for different absorber lengths $L_{\text {abs }}$ where $S_{\text {pn }}=0.4 \mu \mathrm{m}$ is fixed. A responsivity of $>1 \mathrm{~A} / \mathrm{W}$ is expected for $L_{\mathrm{abs}} \geq 4 a(1.68 \mu \mathrm{m})$. A decrease in $L_{\text {abs }}$ degrades the absorption efficiency, but still maintains $>0.5 \mathrm{~A} / \mathrm{W}$ for $L_{\mathrm{abs}} \leq 2 a(0.84 \mu \mathrm{m})$. As well as the capacitance simulation, these results clearly promise the highly-efficient ultrasmall PD towards configuring an amplifier-less receiver.

\section{Fabrication and Measurement of PhC PD}

\subsection{Device Preparation}

For device fabrication, we employed the same process for combining a $\mathrm{PhC}$ waveguide and an ultrasmall $\mathrm{BH}$ as used that used for $\mathrm{PhC}$ nanolasers and all-optical memories [9], [11]. An ultra-small InGaAs absorber layer, which was lattice matched to InP, was embedded in a line-defect $\mathrm{PhC}$ waveguide using butt-joint techniques. Figure 4 (a) shows a SEM image of the PhC PD, revealing a flat surface resulting from the successful butt-joint regrowth. PhC air holes were formed by electron-beam lithography and $\mathrm{Cl}_{2}$ based dry etching. A lateral p-i-n junction was formed by employing $\mathrm{Zn}$ diffusion and $\mathrm{Si}$ ion implantation for the $\mathrm{p}$ and n-type doping, respectively. After metallization, a sacrificial layer was etched to form an air-bridge structure. As mentioned later, InGaAs or InAlAs was employed for the sacrificial layer, and the latter can work to reduce the dark current in operation.

We measured the device characteristics by employing a wavelength-tunable laser light, as shown in Fig. 4 (a). The (a)

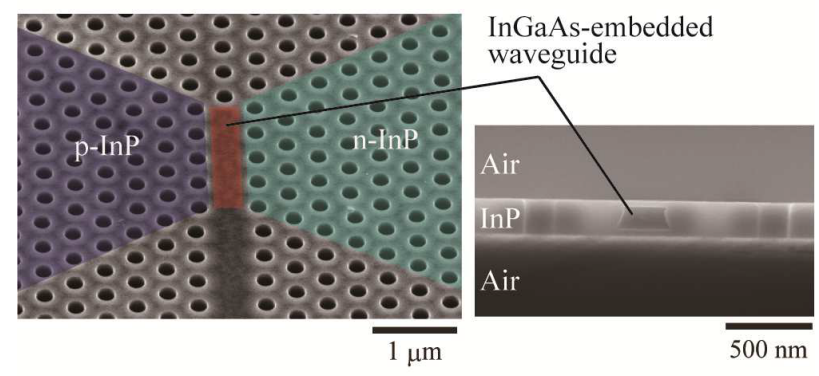

(b)

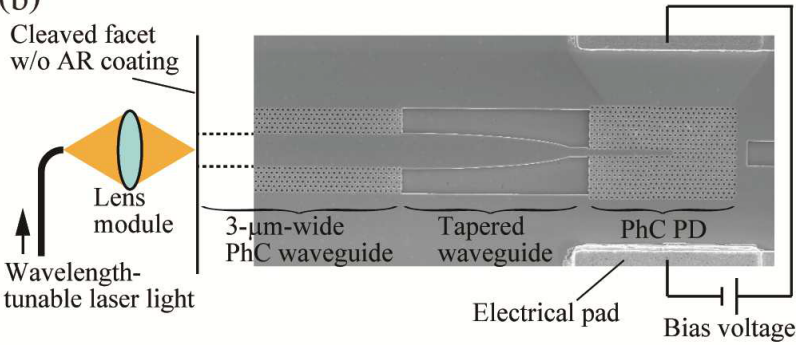

Fig. 4 Fabricated sample and measurement setup. (a) SEM images of top and cross-section. (b) Measurement setup and input waveguides for injecting light into the PD.

light was injected into the $\mathrm{InP} \mathrm{PhC}$ waveguide from the fiber via a lens module, a $3-\mu \mathrm{m}$-wide $\mathrm{PhC}$ waveguide, and a tapered waveguide. In the measurement, a fiber polarization controller was used to tune the input light to TE polarization. The total coupling loss from the fiber to the input $\mathrm{PhC}$ waveguide was $11 \mathrm{~dB}$, which was used to estimate the onchip power.

\subsection{Static Responses}

The photocurrent $(I)$ was measured for a different bias voltage $(V)$ and $\mathrm{CW}$ optical power. For the first trial device fabrication, an InGaAs sacrificial layer was employed. As shown in Fig. 5 (a), the dark currents were approximately 3 and $40 \mathrm{nA}$ when the reverse bias voltages were set at -2 and $-4 \mathrm{~V}$, respectively. This originated from the leakage current that passed through the InGaAs sacrificial layer and the surface of the InP buffer layer, despite the formation of an air-bridge structure. To solve this, the sacrificial layer was changed to InAlAs, which has a large bandgap energy, and an Fe-doped InP buffer layer was employed to form the semi-insulating surface. As a result, the dark current in the same voltage range fell to less than $100 \mathrm{pA}$, as shown in Fig. 5 (b).

Figure 5 (c) shows photocurrent as a function of optical input power at a bias voltage of $-2 \mathrm{~V}$ for a device with an InAlAs sacrificial layer. We successfully estimated a large optical responsivity of $0.98 \mathrm{~A} / \mathrm{W}$ even for $L_{\mathrm{abs}}=1.7 \mu \mathrm{m}$. Specifically, our BH formation does not increase the nonradiative carrier recombination loss thanks to the successful butt-joint epitaxial growth. In fact, a carrier lifetime of $7 \mathrm{~ns}$ has been confirmed for our BH structure [11], and this would 
(a)
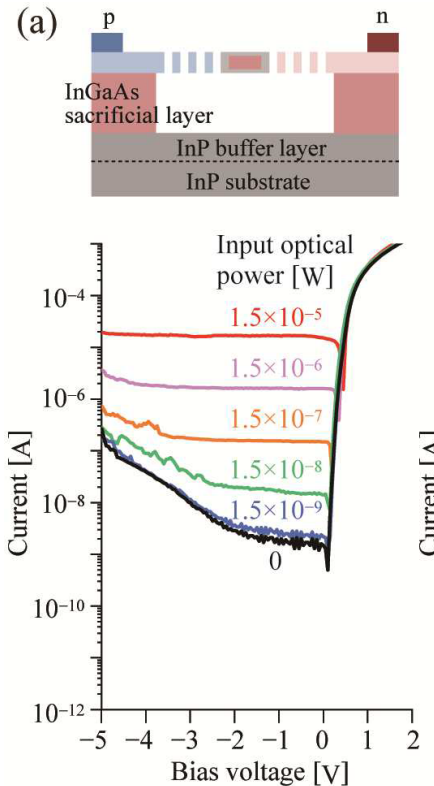

(b)
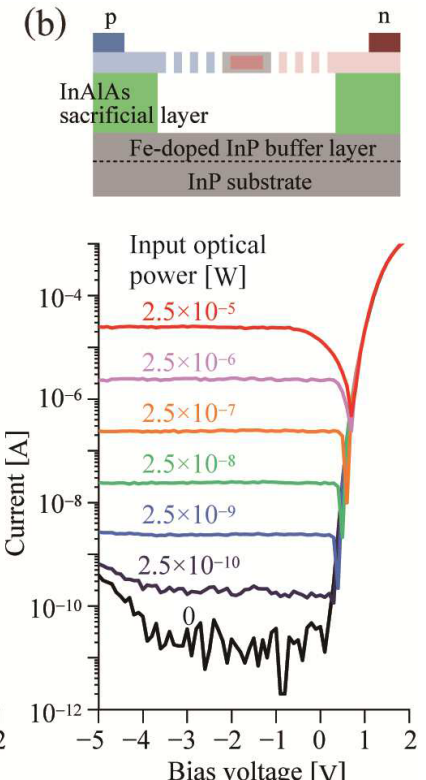

(c)

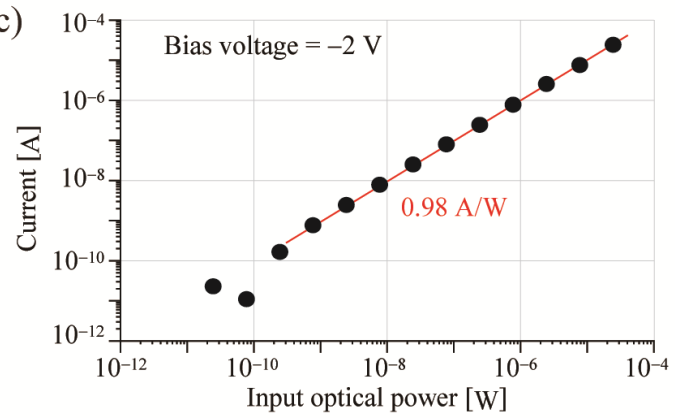

(d)

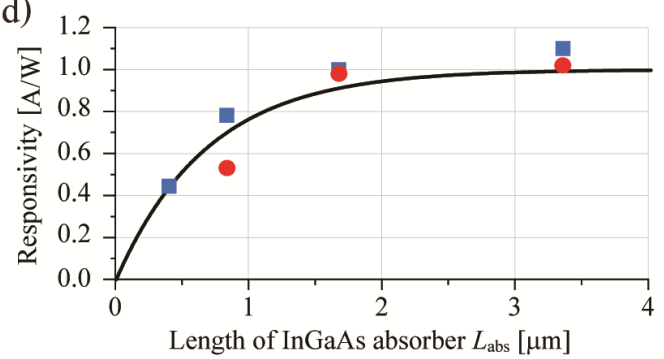

Fig.5 Static responses for CW light input. (a) DC photocurrent versus bias voltage characteristics for PD with InGaAs sacrificial layer. (b) PD with InAlAs sacrificial layer. The absorber length is $L_{\mathrm{abs}}=1.7 \mu \mathrm{m}$ for both (a) and (b). The light wavelength is $1536.7 \mathrm{~nm}$. (c) Photocurrent versus optical power characteristics for PD with InAlAs sacrificial layer. Inset shows the photocurrent spectrum for the optical power of $10 \mu \mathrm{W}$. (d) DC responsivity versus $L_{\mathrm{abs}}$ characteristics. Experimental results (red circles), simulated results of Fig. 3 (e) (blue squares), and theoretical curve of Eq. (3) (black curve) are shown.

be long enough to prevent carrier loss during a fast carrier extraction. The inset of Fig. 5 (c) shows the photocurrent spectrum, which shows that the high and flat responsivity of more than $50 \mathrm{~nm}(1490-1540 \mathrm{~nm})$ was observed.

The photocurrent was reduced when the absorber length became short, as summarized in Fig. 5 (d). The theoretical responsivity $\eta_{\mathrm{PD}}$ for a single roundtrip of light in the

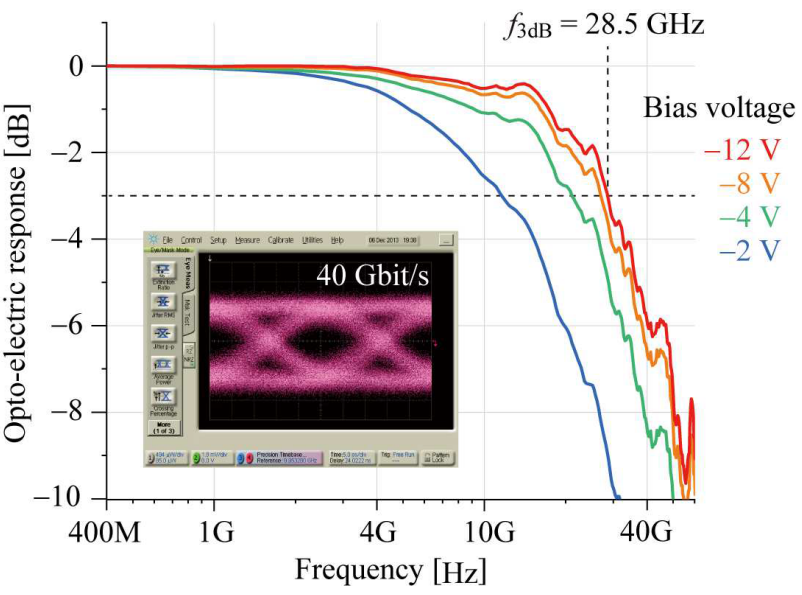

Fig. 6 Small signal responses for different reverse-bias voltages. Inset shows the eye diagram for $40 \mathrm{Gbit} / \mathrm{s} \mathrm{NRZ} \mathrm{optical} \mathrm{signals.} \mathrm{The} \mathrm{input} \mathrm{wave-}$ length was $1536.7 \mathrm{~nm}$ and the optical peak power was $100 \mu \mathrm{W}$.

absorber is given by

$$
\eta_{\mathrm{PD}}=\eta_{\mathrm{eff}} \cdot \frac{e}{h v} \cdot\left\{1-\exp \left(-2 \frac{n_{\mathrm{g}}}{n} \alpha_{\mathrm{abs}} \Gamma L_{\mathrm{abs}}\right)\right\}
$$

where $e$ is the electron charge, $h$ is the Planck constant, $v$ is the frequency of light, $n$ is the material index, $n_{\mathrm{g}}$ is the group index, $\alpha_{\text {abs }}$ is the absorption constant of InGaAs, $\Gamma$ is the optical confinement factor, and $L_{\mathrm{abs}}$ is the absorber length. $\eta_{\mathrm{eff}}$ is a loss factor that includes the losses for both light and the photo-generated carrier. Figure 5 (d) includes the theoretical curves given by Eq. (4), in which we adopted the simulated values of $\Gamma=0.5$ and $n_{\mathrm{g}}=5$, and assumed parameters of $\alpha_{\mathrm{abs}}=10^{4} \mathrm{~cm}^{-1}, n=3.4$, and $\eta_{\mathrm{eff}}=0.8$. The theoretical curve is a good fit with the experimental plots and the simulation results estimated from Fig. 3 (e). We confirmed that the shortest length with which to maintain a high responsivity close to $1 \mathrm{~A} / \mathrm{W}$ was $L_{\mathrm{abs}}=1.7 \mu \mathrm{m}$.

\subsection{Dynamic Responses}

We investigated the dynamic performance of our PD by injecting an intensity-modulated optical signal under a reverse bias through a bias tee thereby extracting an RF signal. Figure 6 shows the operation dynamics of our PD, into which we injected an intensity-modulated optical signal with a peak power of $100 \mu \mathrm{W}$. At the low frequency region $(<1 \mathrm{GHz})$, the bias voltage as low as $-2 \mathrm{~V}$ works for the high responsivity, which suggests that the carrier drift is sufficiently faster than the carrier recombination. The maximum 3-dB bandwidth was $28.5 \mathrm{GHz}$ when the bias voltage was $-12 \mathrm{~V}$, which reveals the capability for a bit rate of around $50 \mathrm{Gbit} / \mathrm{s}$ for a non-return-to-zero (NRZ) signal. Actually, the clear eye opening was observed for 40-Gbit/s NRZ signals generated with a $2^{31}-1$ pseudo-random bit sequence, as shown in the inset of Fig. 6. The high bias voltage $(-12 \mathrm{~V})$ required for the high frequency region is probably caused from the wider depletion region than we expected. The wide depletion region degrades the internal 
electric field, and therefore the bias voltage should be increased to maintain the fast carrier extraction. This bias voltage will be reduced by optimizing the separation between $p$ and $\mathrm{n}$ region, as suggested in the simulation of Fig. 3 (d).

\section{Resistor-Loaded PhC PD}

\subsection{Device Preparation and Electro-Optic Probing Setup}

The ultrasmall capacitance of our PD enables us to connect it with a high load resistance without the need for amplifiers to convert photocurrent to voltage while maintaining a large RC bandwidth. However, there has been no report evaluating the on-chip light-to-voltage conversion dynamics of resistor-loaded nano-PDs. The experimental difficulty is that a conventional measurement using an oscilloscope/network analyzer with an additional electrical pad would hinder correct device evaluation, because in most cases their impedances are lower than the device load of $50 \Omega$. This makes it difficult to measure the voltage across the load. (Note that direct connection with a highimpedance CMOS gate would be available for a photoreceiver in an on-chip-communication application.) In our measurement, we employed an electro-optic (EO) probing technique [23]. When we prepared the sample for EO probing, our PhC-PD was connected to a load resistor $R_{\text {load }}$ with a gold strip line with a length $L_{\text {strip }}$, as shown in Fig. 7 (a).

The experimental setup for EO probing is shown in Fig. 7 (b). Sinusoidal modulated light $(50 \mathrm{MHz})$ was injected into the PhC-PD. Photocurrent flows into the load resistor, and generates a modulated electric field (proportional to the voltage) between the strip lines. An EO probe consisting of an optical fiber with an EO crystal (ZnTe) was brought towards the strip line. CW light $(\lambda=1.55 \mu \mathrm{m})$ was separately injected into the EO probe and sensed the modulated electric field via the EO crystal. By combining a polarization beam splitter and a balanced photoreceiver, the polarization change of the $\mathrm{CW}$ light was detected as an EO probing voltage. Before the device measurement, the EO probing voltage for $\mathrm{AC}$ voltage applied to the strip line was acquired to obtain the correspondence between the two voltages. To this end, a sinusoidal voltage signal from a function generator was directly applied to the reference strip line and the EO-probing voltage was detected at the mid-point between the strip lines. As shown in Fig. 7 (c), we observed a clear proportional relationship with the minimum observable voltage across the strip line of $40 \mathrm{mV}$. Thereafter, we replaced the reference with a PhC-PD sample to evaluate the photo-generated voltage.

\subsection{Demonstration of Light-to-Voltage Conversion}

Figure 8 (a) shows the light-to-voltage conversion efficiency $\eta_{\mathrm{LV}}$ for PDs with different $R_{\text {load }}$ values. The generated AC voltage $V_{\mathrm{pp}}$ clearly increased as $R_{\text {load }}$ became larger. A maximum $\eta_{\mathrm{LV}}=3.95 \mathrm{kV} / \mathrm{W}$ was achieved for $R_{\text {load }}=8.8 \mathrm{k} \Omega$. (a)
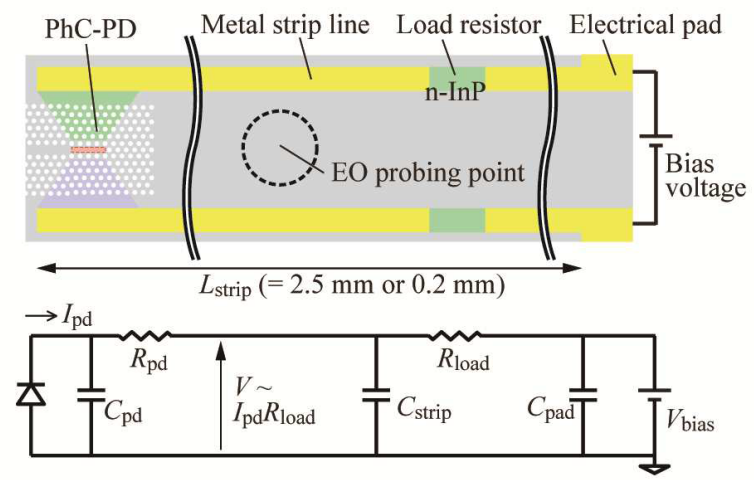

(b)

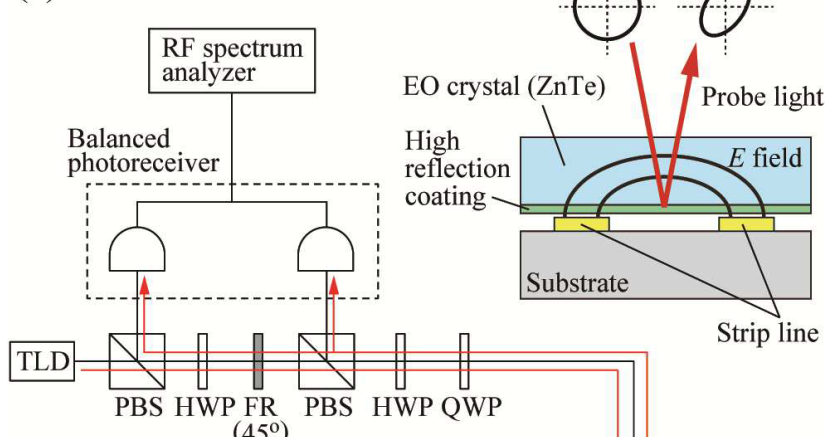
$\left(45^{\circ}\right)$

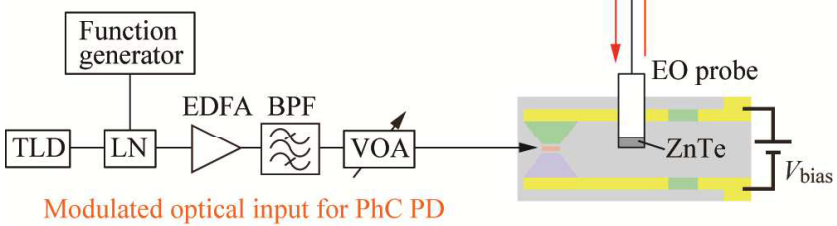

(c)

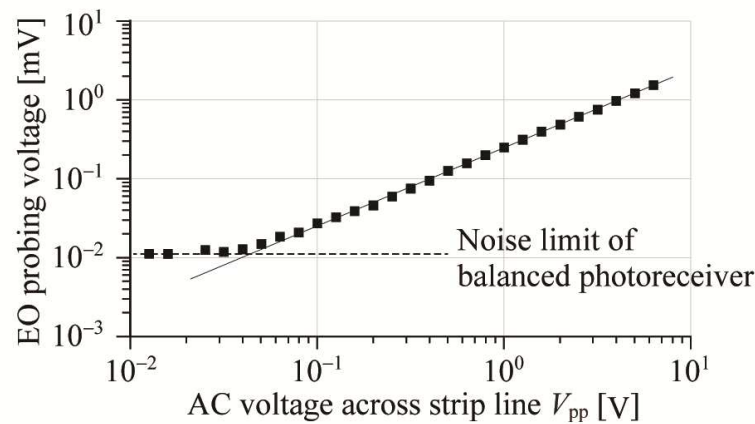

Fig. 7 Resistor-loaded PhC-PD and EO probing measurement setup. (a) Schematic of sample (top) and corresponding equivalent circuit (bottom). (b) Experimental setup for EO probing measurement. (TLD: Tunable laser diode, LN: Lithium-niobate modulator, EDFA: Erbium-doped fiber amplifier, BPF: Band-pass filter, VOA: Variable optical attenuator, PBS: Polarization beam splitter, HWP: Half-wave plate, QWP: Quarterwave plate, FR: Faraday rotator) (c) EO probing voltage for AC voltage applied to the reference strip line. 
(a)

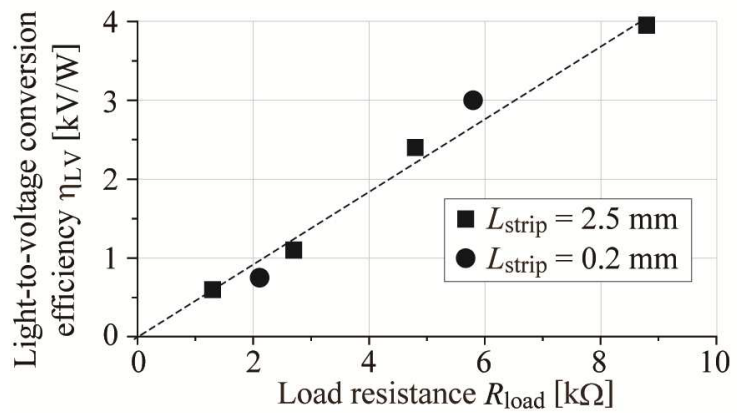

(b)

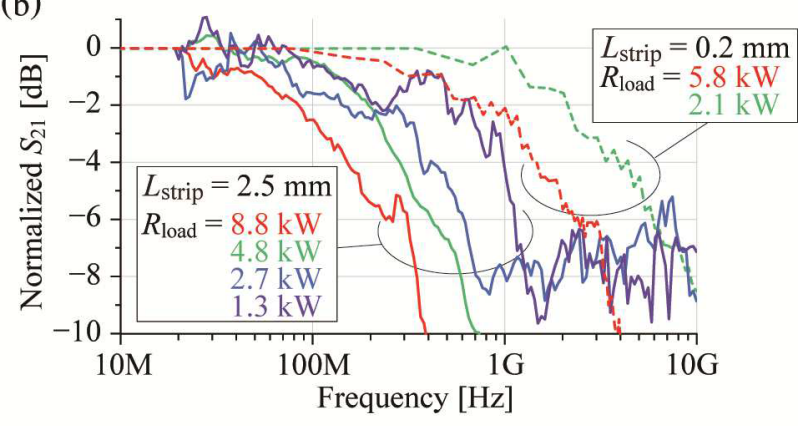

(c)

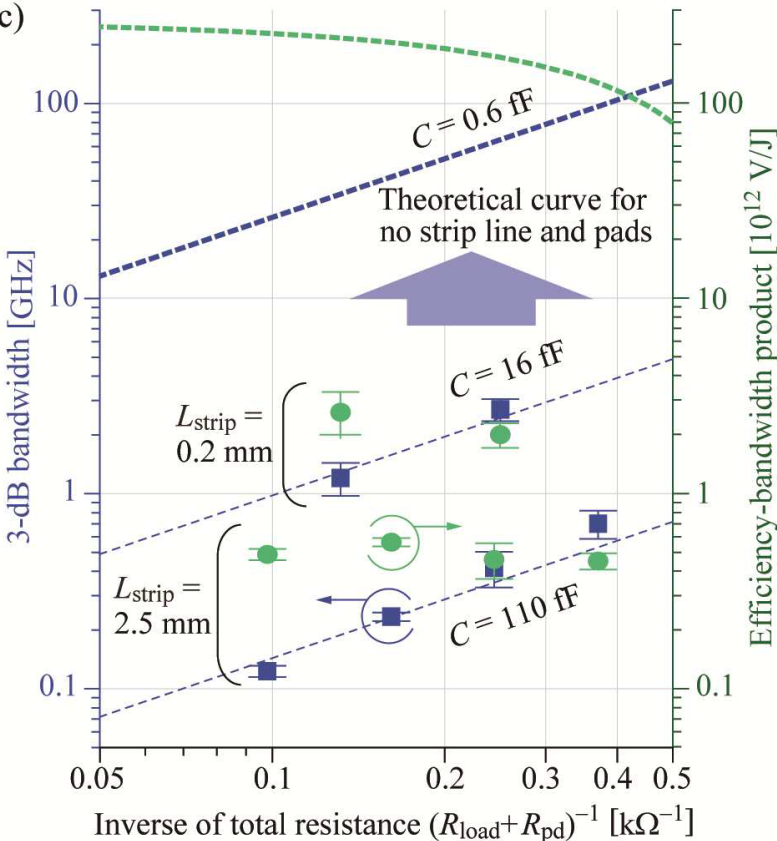

Fig. 8 Measurement results for voltage generation in resistor-loaded PhC-PD. (a) Light-to-voltage conversion efficiency for different load resistances $R_{\text {load }}$. Square and circle plots show results for $L_{\text {strip }}$ values of 2.5 and $0.2 \mathrm{~mm}$, respectively. (b) Small-signal responses for different load resistances $R_{\text {load }}$ and strip line lengths $L_{\text {strip }}$. (c) 3-dB bandwidth (square plots for left axis) and efficiency-bandwidth product (circle plots for right axis). The plots show the experimental results, and the dashed curves show the calculated results considering both the PD junction capacitance and the parasitic capacitances. The bold dashed curves are calculated under the assumption of no parasitic capacitances.
These results show that an optical power of $50 \mu \mathrm{W}$ can generate the $V_{\mathrm{pp}}$ of $200 \mathrm{mV}$ that is required for a CMOS inverter [5]. On the other hand, the operation bandwidth is limited by RC, although a gold strip line and a pad with a much larger capacitance than the PhC-PD were included in our sample because they were necessary for EO probing. Figure 8 (a) shows the frequency responses for different $R_{\text {load }}$ values, in which samples with different $L_{\text {strip }}$ values of 2.5 and $0.2 \mathrm{~mm}$ were evaluated. The smaller $R_{\text {load }}$ and $L_{\text {strip }}$, which resulted in a shorter RC time, appear to increase the bandwidth. The RC-limited bandwidth for $L_{\text {strip }}=2.5 \mathrm{~mm}$ was estimated from $f_{\mathrm{RC}}=120-750 \mathrm{MHz}$ for $R_{\text {load }}$ values of $1.3-8.8 \mathrm{k} \Omega$, while that for $L_{\text {strip }}=0.2 \mathrm{~mm}$ increased to $f_{\mathrm{RC}}=1.2-2.7 \mathrm{GHz}$. Figure 8 (c) summarizes the $3-\mathrm{dB}$ bandwidth (blue plots for the left vertical axis) as a function of $1 /\left(R_{\text {load }}+R_{\text {pd }}\right)$. These plots have a linear relation as they are mainly determined by $f_{\mathrm{RC}}=\left[2 \pi\left(R_{\mathrm{pd}}+R_{\text {load }}\right) C\right]^{-1}$, where $C$ consists of both the PhC-PD capacitance and the parasitic capacitance caused by the strip line and pads. The dashed lines are the theoretical curves obtained by assuming $C=16$ and $110 \mathrm{fF}$, which are dominated by parasites, and the fit well with the experimental plots.

Another figure we evaluated was the product of $\eta_{\mathrm{LV}}$ and $f_{\mathrm{RC}}$, which are in a trade-off relationship, because they are proportional to $R_{\text {load }}$ and $\left(R_{\mathrm{pd}}+R_{\text {load }}\right)^{-1}$, respectively. This efficiency-bandwidth product (EBP) [V/W.Hz] $(=[\mathrm{V} / \mathrm{J}])$ can indicate the optical energy needed to generate the required voltage, regardless of the bit rate of the optical signal. The EBPs are denoted by green plots on the right vertical axis in Fig. 8 (d). A shorter $L_{\text {strip }}$ enhances the EBP because $f_{\mathrm{RC}}$ increases while $\eta_{\mathrm{LV}}$ remains constant (See Fig. 8 (a)). The EBP values were in the $4-5 \times 10^{11}$ and $2-3 \times$ $10^{12} \mathrm{~V} / \mathrm{J}$ ranges for $L_{\text {strip }}=2.5$ and $0.2 \mathrm{~mm}$, respectively. As a result, they can be translated to the required optical energies of 200 and $33 \mathrm{fJ} /$ bit for $L_{\text {strip }}=2.5$ and $0.2 \mathrm{~mm}$, respectively, to obtain $V_{\mathrm{pp}}=200 \mathrm{mV}$ with an NRZ optical signal. We also theoretically discuss an ideal case where there is no parasitic capacitance. The bold dashed curves in Fig. 8 (d) denote $f_{\mathrm{RC}}$ and EBP in an ideal situation calculated by assuming only a PD junction capacitance of $C=0.6 \mathrm{fF}$. This makes the bandwidth higher than $10 \mathrm{GHz}$, which should be practically acceptable. Subsequently, the expected EBP exceeds $10^{14} \mathrm{~V} / \mathrm{J}$, corresponding to a required optical energy of less than $1 \mathrm{fJ} / \mathrm{bit}$. These performances promise to surpass the performance of a conventional PD-TIA circuit. Such a situation can be realized by removing the strip line and pads used in the experiment, because they were simply needed for the EO-probing measurement. Our experimental and theoretical results for an ultrasmall PhC-PD have revealed the feasibility of an amplifier-less photoreceiver on a chip with a practically acceptable size, efficiency, bandwidth, and power consumption.

\section{Conclusions}

Ultralow capacitance PDs offer the opportunity to configure a resistor-loaded photoreceiver that does not require an 
amplifier. To this end, we employed a $\mathrm{PhC}$ nanostructure in which we embedded a small InGaAs absorber, which allowed us to reduce the detector length to just $1.7 \mu \mathrm{m}$ while demonstrating a high responsivity of $1 \mathrm{~A} / \mathrm{W}$ and eye opening for a $40 \mathrm{Gbit} / \mathrm{s}$ signal. The theoretical device capacitance fell to less than $1 \mathrm{fF}$. We fabricated a resistor-loaded PhC$\mathrm{PD}$, and successfully demonstrated light-to-voltage conversion with an efficiency of up to $4 \mathrm{kV} / \mathrm{W}$, which is comparable to recently available TIA-integrated photoreceivers. The $\mathrm{GHz}$ level operation bandwidth was also evaluated, and it would be enhanced simply by removing the parasitic elements and thus increasing the RC bandwidth above $10 \mathrm{GHz}$. These demonstrations clearly revealed a promising way of realizing a photoreceiver that operates with an optical energy of less than $1 \mathrm{fJ} /$ bit without any amplifier energy. Such a receiver will enable us to develop a photonic network on chip architecture with an unprecedentedly high density.

\section{Acknowledgments}

This work was supported by CREST (JPMJCR15N4), Japan Science and Technology Agency.

We thank T. Tamamura, H. Onji, Y. Shouji, and K. Ishibashi for support in fabricating the device. We also thank H. Togo for support in establishing the EO-probing measurement setup.

\section{References}

[1] M. Notomi, K. Nozaki, A. Shinya, S. Matsuo, and E. Kuramochi, "Toward fJ/bit optical communication in a chip," Optics Communications, vol.314, pp.3-17, 2014.

[2] C. Sun, M.T. Wade, Y. Lee, J.S. Orcutt, L. Alloatti, M.S. Georgas, A.S. Waterman, J.M. Shainline, R.R. Avizienis, S. Lin, B.R. Moss, R. Kumar, F. Pavanello, A.H. Atabaki, H.M. Cook, A.J. Ou, J.C. Leu, Y.-H. Chen, K. Asanović, R.J. Ram, M.A. Popović, and V.M. Stojanović, "Single-chip microprocessor that communicates directly using light," Nature, vol.528, no.7583, pp.534-538, 2015.

[3] Y.A. Vlasov, "Silicon CMOS-Integrated Nano-Photonics for Computer and Data Communications Beyond 100G," IEEE Commun. Mag., vol.50, no.2, pp.S67-S72, 2012.

[4] D. Okamoto, Y. Suzuki, K. Yashiki, Y. Hagihara, M. Tokushima, J. Fujikata, M. Kurihara, J. Tsuchida, T. Nedachi, J. Inasaka, and K. Kurata, "A 25-Gb/s 5 x 5 mm(2) Chip-Scale Silicon-Photonic Receiver Integrated With 28-nm CMOS Transimpedance Amplifier," J. Lightwave. Technol., vol.34, no.12, pp.2988-2995, 2016.

[5] S. Assefa, F.N. Xia, W.M.J. Green, C.L. Schow, A.V. Rylyakov, and Y.A. Vlasov, "CMOS-Integrated Optical Receivers for On-Chip Interconnects," IEEE J. Select. Topics Quantum Electron., vol.16, no. 5, pp.1376-1385, 2010.

[6] X.Z. Zheng, D. Patil, J. Lexau, F. Liu, G.L. Li, H. Thacker, Y. Luo, I. Shubin, J.D. Li, J. Yao, P. Dong, D.Z. Feng, M. Asghari, T. Pinguet, A. Mekis, P. Amberg, M. Dayringer, J. Gainsley, H.F. Moghadam, E. Alon, K. Raj, R. Ho, J.E. Cunningham, and A.V. Krishnamoorthy, "Ultra-efficient $10 \mathrm{~Gb} / \mathrm{s}$ hybrid integrated silicon photonic transmitter and receiver," Opt. Express, vol.19, no.6, pp.5172-5186, 2011.

[7] D.A.B. Miller, "Device requirements for optical interconnects to silicon chips," Proc. IEEE, vol.97, no.7, pp.1166-1185, 2009.

[8] C. Debaes, A. Bhatnagar, D. Agarwal, R. Chen, G.A. Keeler, N.C. Helman, H. Thienpont, and D.A.B. Miller, "Receiver-less optical clock injection for clock distribution networks," IEEE J. Select. Topics Quantum Electron., vol.9, no.2, pp.400-409, 2003.
[9] S. Matsuo, A. Shinya, T. Kakitsuka, K. Nozaki, T. Segawa, T. Sato, Y. Kawaguchi, and M. Notomi, "High-speed ultracompact buried heterostructure photonic-crystal laser with $13 \mathrm{fJ}$ of energy consumed per bit transmitted," Nature Photonics, vol.4, no.9, pp.648-654, 2010.

[10] K. Nozaki, S. Matsuo, T. Fujii, K. Takeda, M. Ono, A. Shakoor, E. Kuramochi, and M. Notomi, "Photonic-crystal nano-photodetector with ultrasmall capacitance for on-chip light-to-voltage conversion without an amplifier," Optica, vol.3, no.5, pp.483-492, 2016.

[11] K. Nozaki, A. Shinya, S. Matsuo, Y. Suzaki, T. Segawa, T. Sato, Y. Kawaguchi, R. Takahashi, and M. Notomi, "Ultralow-power alloptical RAM based on nanocavities," Nature Photonics, vol.6, no.4, pp. 248-252, 2012.

[12] J. Joo, K.-S. Jang, S.H. Kim, I.G. Kim, J.H. Oh, S.A. Kim, G.-S. Jeong, Y. Kim, J.-E. Park, S. Kim, H. Chi, D.-K. Jeong, and G. Kim, "Silicon photonic receiver and transmitter operating up to 36 $\mathrm{Gb} / \mathrm{s}$ for lambda similar to $1550 \mathrm{~nm}$," Optics Express, vol.23, no.9, pp.12232-12243, 2015.

[13] T. Takemoto, H. Yamashita, F. Yuki, N. Masuda, H. Toyoda, N. Chujo, Y. Lee, S. Tsuji, and S. Nishimura, "A 25-Gb/s 2.2-W 65-nm CMOS Optical Transceiver Using a Power-Supply-VariationTolerant Analog Front End and Data-Format Conversion," IEEE J. Solid-State Circuits, vol.49, no.2, pp.471-485, 2014.

[14] H. Fukuyama, K. Sano, K. Murata, H. Kitabayashi, Y. Yamane, T. Enoki, and H. Sugahara, "Photoreceiver module using an InP HEMT transimpedance amplifier for over $40 \mathrm{~Gb} / \mathrm{s}$," IEEE J. Solid-State Circuits, vol.39, no.10, pp.1690-1696, 2004.

[15] A. Huber, D. Huber, T. Morf, H. Jäckel, C. Bergamaschi, V. Hurm, M. Ludwig, and M. Schlechtweg, "Monolithic, high transimpedance gain $(3.3 \mathrm{k} \Omega$ ), 40 Gbit s InP-HBT photoreceiver with differential outputs," Electron. Lett., vol.35, no.11, pp.897-898, 1999.

[16] K. Takahata, Y. Muramoto, H. Fukano, and Y. Matsuoka, " $52 \mathrm{GHz}$ bandwidth monolithically integrated WGPD/HEMT photoreceiver with large $\mathrm{O} / \mathrm{E}$ conversion factor of $105 \mathrm{~V} / \mathrm{W}$," Electron. Lett., vol.35, no.19, pp.1639-1640, 1999.

[17] Finisar. Available: https://www.finisar.com/optical-components/ xprv2022a

[18] R. Going, T.J. Seok, J. Loo, K. Hsu, and M.C. Wu, "Germanium wrap-around photodetectors on Silicon photonics," Opt. Express., vol.23, no.9, pp.11975-11984, 2015.

[19] L. Virot, P. Crozat, J.-M. Fédéli, J.-M. Hartmann, D. Marris-Morini, E. Cassan, F. Boeuf, and L. Vivien, "Germanium avalanche receiver for low power interconnects," Nature Communications, vol.5, p.4957, 2014.

[20] K. Takeda, T. Sato, A. Shinya, K. Nozaki, W. Kobayashi, H. Taniyama, M. Notomi, K. Hasebe, T. Kakitsuka, and S. Matsuo, "Few-fJ/bit data transmissions using directly modulated lambdascale embedded active region photonic-crystal lasers," Nature Photonics, vol.7, no.7, pp.569-575, 2013.

[21] A. Shakoor, K. Nozaki, E. Kuramochi, K. Nishiguchi, A. Shinya, and M. Notomi, "Compact 1D-silicon photonic crystal electro-optic modulator operating with ultra-low switching voltage and energy," Optics Express, vol.22, no.23, pp.28623-28634, 2014.

[22] Lumerical. Available: https://www.lumerical.com/

[23] T. Nagatsuma, "Measurement of High-Speed Devices and Integrated-Circuits Using Electrooptic Sampling Technique," IEICE Trans. Electron., vol.E76c, pp.55-63, 1993. 


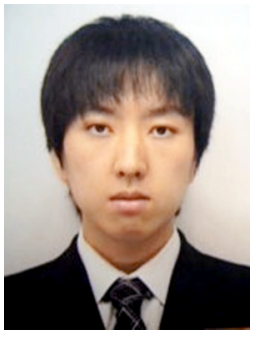

Kengo Nozaki received the B.E., M.E., and $\mathrm{Ph} . \mathrm{D}$. degrees in electrical and computer engineering from Yokohama National University, Kanagawa, in 2003, 2005, and 2007, respectively. He joined NTT Basic Research Laboratories in 2008. His current interests are alloptical switches, memories, and electro-optic devices based on photonic crystals and related photonic nanostructures. He received the Best Paper Award from Photonics in switching (PS) in 2012, IEICE Electronics Society Young Researchers Award in 2014, and the Best Paper Award from OECC/PS in 2016. Dr. Nozaki is a member of the Japan Society of Applied Physics (JSAP).

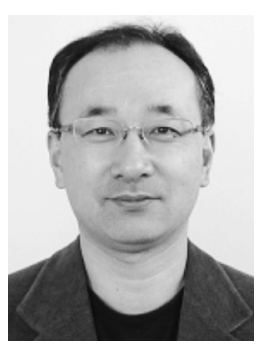

Shinji Matsuo received the B.E. and M.E. degrees in electrical engineering from Hiroshima University, Hiroshima, Japan, in 1986 and 1988, and the Ph.D. degree in electronics and applied physics from Tokyo Institute of Technology, Tokyo, Japan, in 2008. In 1988, he joined NTT Opto-electronics Laboratories, Atsugi, where he was engaged in research on photonic functional devices using MQW-pin modulators and VCSELs. In 1997, he researched optical networks using WDM technologies at NTT Network Innovation Laboratories, Yokosuka. Since 2000, he has been researching high-speed tunable optical filters and lasers for photonic packet switching at NTT Photonics Laboratories, Atsugi. Dr. Matsuo is a member of the IEEE Photonics Society, Japan Society of Applied Physics and the Institute of Electronics, Information and Communication Engineers (IEICE) of Japan.

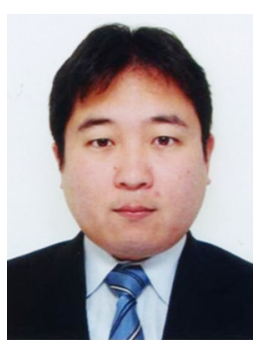

Koji Takeda received the B.S., M.S., and $\mathrm{Ph} . \mathrm{D}$. degrees in electronics engineering from the University of Tokyo, Tokyo, Japan, in 2005, 2007, and 2010, respectively. From 2008 to 2010, he received research fellowship for young scientists from Japan Society for the Promotion of Science. He joined NTT Photonics Laboratories in 2010. His current research interests include ultralow-power optical interconnect, InP photonic integrated circuit, and photonic crystal lasers. He received the Best Student Paper Award from IEEE Photonics Society in 2009, and the Outstanding Student Presentation Award from JSAP in 2010. Dr. Takeda is a member of the IEEE Photonics Society, Japan Society of Applied Physics (JSAP), and the Institute of Electronics, Information and Communication Engineers (IEICE).

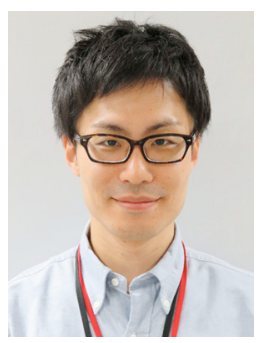

Takuro Fujii received the B.E. and M.E. degrees in system design engineering from Keio University, Kanagawa, in 2010 and 2012. He joined NTT Photonics Laboratories in 2012. He has been researching MOVPE growth of III-V semiconductors and the development of III-V semiconductor lasers on $\mathrm{Si}$ for photonic integrated circuits. He received the Young Scientist Presentation Award from the JSAP in 2014. He is a member of JSAP.

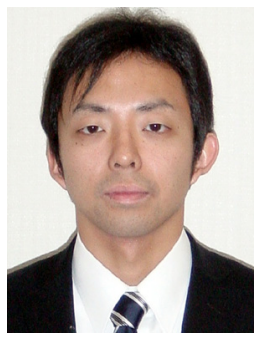

Masaaki Ono received the B.E., M.E., and $\mathrm{Ph} . \mathrm{D}$. degrees in electronic engineering from Tohoku University, Miyagi, in 2007, 2008, and 2011, respectively. He joined NTT Basic Research Laboratories in 2011. His current interests are optical devices based on plasmonic structures and nanostructure fabrication. Dr. Ono is a member of the Japan Society of Applied Physics (JSAP).

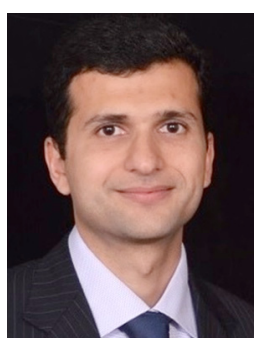

Abdul Shakoor received B.E in Avionics from National University of Science and Technology (NUST), Pakistan in 2004, European Commission funded Erasmus Mundus joint Masters in Photonics from Royal Institute of Technology (KTH), Sweden and University of St Andrews, Scotland in 2008 and $\mathrm{PhD}$ from University of St Andrews in 2013. His main interest is developing novel integrated photonic devices for telecommunication and sensing applications. Currently, he is working as a senior research fellow at Optoelectronics Research Centre (ORC) at the University of Southampton. Prior to joining ORC, he worked as a research associate at University of Glasgow and Basic Research Laboratories of Nippon Telegraph and Telephone Corporation (NTT-BRL). His research highlights include development of electrically pumped on-chip silicon nano light source operating at telecommunication wavelengths with high power spectral density, development of silicon electro-optic modulator operating with sub $\mathrm{fJ} /$ bit switching energy and monolithic integration of nanophotonic structures with CMOS detectors for sensing applications. Dr Shakoor is a member of Optical Society of America (OSA).

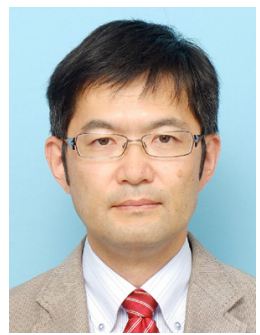

Eiichi Kuramochi received the B.E., M.E., and $\mathrm{Ph}$. D degrees in electrical engineering from Waseda University, Tokyo in 1989, 1991, and 2004, respectively. In 1991, he joined NTT Optoelectronics Laboratories, where he was engaged in research on semiconductor nanostructures for photonic devices. In 1998, he moved to NTT Basic Research Laboratories. He has been engaged in research on photonic crystals. Dr. Kuramochi is a member of the IEEE Photonics Society and the Japan Society of Applied

Physics.

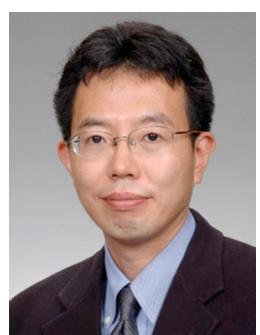

Masaya Notomi received B.E., M.E. and $\mathrm{Ph}$. D. degrees in applied physics from University of Tokyo, Japan in 1986, 1988, and 1997, respectively. In 1988, he joined NTT. Since then, his research interest has been to control the optical properties of materials/devices by artificial nanostructures (quantum wires/dots and photonic crystals). He is a group leader of Photonic Nanostructure Research Group and Senior Distinguished Scientist in NTT Basic Research Laboratories. He is also a guest professor of Tokyo Institute of Technology. He received IEEE/LEOS Distinguished Lecturer Award (2006), JSPS prize (2009), Japan Academy Medal (2009), and The Commendation by Japanese Minister of Education (2010). He is serving as a member of National University Corporation Evaluation Committee in the Japanese government. Dr. Notomi is a member of the Japan Society of Applied Physics, American Physical Society (APS), IEEE, and Optical Society of America (OSA). 